\title{
Classification of Loratadine Based on the Biopharmaceutics Drug Classification Concept and Possible in Vitro-in Vivo Correlation
}

\author{
M. Zahirul I. Khan, ${ }^{*}$ Dragica RauŠL, Ružica ZANošKI, Snježana ZIDAR, Jasna Horvat MiKulčIĆ, ${ }^{1)}$ \\ Lara Krizmanić, Maja EšKinja, ${ }^{2)}$ Boris MiLdner, and Zdravka KneŽević
}

PLIVA Research Institute Ltd.; Prilaz baruna Filipovića 29, 10000 Zagreb, Croatia.

Received February 13, 2004; accepted June 7, 2004

Loratadine was studied both in vitro and in vivo (in healthy humans) to classify it according to the Biopharmaceutics Classification System (BCS) in order to gain more understanding of the reasons for its highly variable nature with respect to plasma time profiles, and to determine the most appropriate dissolution test conditions for in vitro assessment of the release profile of the drug from solid dose forms. Based on the solubility of loratadine determined under various $\mathrm{pH}$ conditions and its permeability through Caco-2 monolayers, loratadine was classified as a Class II drug. Plasma profiles were predicted by convolution analysis using dissolution profiles obtained under various $\mathrm{pH}$ and hydrodynamic conditions as the input function and plasma time data obtained from a syrup formulation as the weighting function. The predicted profiles based on dissolution studies done at gastric pH values were in reasonable agreement with the mean bio-data suggesting dissolution testing should be done at gastric pH values. However, the bio-data were highly variable and it is suggested this may be due, at least in part, to high individual gastric $\mathrm{pH}$ variability and dissolution occurring in the intestine on some occasions, and therefore, dissolution testing should also be done in simulated intestinal fluid.

Key words biopharmaceutics classification system; biorelevant dissolution testing; convolution; permeability testing; in vitro-in vivo correlation; IVIVC; loratadine

Loratadine, chemically known as ethyl 4-(8-chloro-5,6-dihydro-11H-benzo[5,6]-cyclohepta[1,2-b]pyridin-11-ylidene)1-piperidinecarboxylate, is a weak base with a $\mathrm{p} K_{\mathrm{a}}$ value of ca. 6 (calculated in our laboratories). It is absorbed rapidly following oral administration in humans giving maximum plasma concentration $\left(C_{\max }\right)$ in about $1-1.5 \mathrm{~h}$ when administered under fasting conditions, but the degree of inter-subject variability in the pharmacokinetic parameters was reported to be very high. The $C_{\max }$ and plasma concentration-time $\left(A U C_{t}\right)$ data obtained following oral administration of $10 \mathrm{mg}$, $20 \mathrm{mg}$, and $40 \mathrm{mg}$ loratadine capsules were found to have a dose-proportional relationship. ${ }^{3)}$

Following oral dosing $80 \%$ of the total dose administered was found equally distributed between urine and faeces in the form of metabolic products within $10 \mathrm{~d}^{4}{ }^{4}$ The kinetics of loratadine observed during a multiple dose study were found comparable to those reported from single dose studies, but the mean plasma concentration-time data were approximately $90 \%$ greater than that reported for single dose data, and the steady state plasma concentrations were apparent by the fifth day of dosing. ${ }^{5)}$

The objective of the studies reported here was two fold; firstly, to classify loratadine according to the biopharmaceutics drug classification scheme, ${ }^{6,7)}$ and secondly, to compare plasma time profiles predicted from in vitro dissolution data with experimental plasma time data in order to select the most appropriate conditions for in vitro assessment of loratadine solid dosage forms. Furthermore, attempts have been made to investigate factors responsible for the high inter-subject variability in pharmacokinetic parameters of the drug.

\section{MATERIALS AND METHODS}

Materials Loratadine of European Pharmacopoeia (EP) quality was purchased from Chemo Iberica S.A. (Lugano, Switzerland). Standard pharmacopoeial grade excipients were used in the tablet formulation. Egg lecithin used for dissolution studies under biorelevant conditions was a gift from Lipoid AG (Cham, Switzerland). Crude sodium taurocholate (batch no. 386645/1, standardised against a lot of the pure substance containing above 97\%) was purchased from Sigma Chemical Co. (St. Louis, U.S.A.). Sodium lauryl sulphate, hydrochloric acid and all other chemicals used for evaluation of the tablets were of analytical grade. The tablets were prepared in-house (by wet granulation technology using starch paste as a binder).

For permeability testing, Caco-2 cells were obtained from the European Collection of Cell Cultures (U.K.) at passage 20. Dulbecco's Eagles medium (DMEM) containing glucose $(4500 \mathrm{mg} / \mathrm{ml})$ was purchased from Imunološki Zavod, Zagreb (Croatia). Fetal bovine serum (FBS), glutamax I, nonessential amino acids (NEAA), penicillin/streptomycin, fungizone and $0.25 \%$ trypsin-EDTA were obtained from Gibco, Life Technologies (U.K.). Hanks balanced salt solution (HBSS) and dimethylsulphoxide (DMSO) were purchased from Sigma-Aldrich (Germany). Tert-butyl methyl ether (TBME) was supplied by Merck (Germany).

Solubility Studies The solubility of loratadine was determined in aqueous solutions with $\mathrm{pH}$ values of 1.2, 2.0, $2.5,3.0,4.5,6.5$ and 7.5 as recommended in regulatory guidelines. ${ }^{7)}$ The media with $\mathrm{pH}$ values of $1.2,2.0,2.5$, and 3.0 were prepared by diluting $\mathrm{HCl}$, whereas $\mathrm{pH} 4.5(0.02 \mathrm{M}$ acetate buffer), 6.5 and 7.5 (both $0.05 \mathrm{M}$ phosphate buffers) were prepared according to USP/NF 19. Saturated solutions of loratadine in specific medium were prepared by dispersing an excessive amount of the drug and then equilibrating at $37^{\circ} \mathrm{C}$ for $24 \mathrm{~h}$ in a shaking bath (medium speed). After equilibrium at room temperature the content was filtered through Vankel Full Flow ${ }^{\mathrm{TM}} 35 \mu \mathrm{m}$ filters and then diluted to measure the loratadine concentration by a UV spectrophotometric method.

Permeability Studies One poorly permeable model 
compound (ranitidine) and one highly permeable model compound (propranolol) were used as markers to rank the level of permeability of loratadine. In one set of experiments the permeability of the two markers and loratadine was tested separately, whereas in another experiment the two markers were tested in a mixture with loratadine.

Cell Culture: Caco- 2 cells were grown in cell culture flasks at $37^{\circ} \mathrm{C}$ with $5 \% \mathrm{CO}_{2}$ under $90 \%$ relative humidity in DMEM supplemented with $10 \%$ FBS, 1\% glutamax I, NEAA, penicillin $(100 \mathrm{U} / \mathrm{ml})$, streptomycin $(100 \mu \mathrm{g} / \mathrm{ml})$, and fungizone $(2.5 \mu \mathrm{g} / \mathrm{ml})$. The cells were detached using trypsin-EDTA when they reached $85-90 \%$ confluence, and at passages 29 and 30 were seeded onto 6-well polycarbonate Costar Transwell ${ }^{\circledR}$ filter inserts with $3 \mu \mathrm{m}$ pores in DMEM at a density of $2.5 \times 10^{5} /$ well. The medium was changed on the following day after seeding, and thereafter, every other day. The quality of the monolayers was monitored by measuring their transepithelial electrical resistance.

Sample Preparation: Loratadine was dissolved in DMSO and then diluted with the transport buffer, HBSS supplemented with $12 \mathrm{~mm}$ HEPES ( $\mathrm{pH} 6.6$ ), to the final concentration of $10 \mu \mathrm{g} / \mathrm{ml}$ and $1 \%$ DMSO. Propranolol and ranitidine were dissolved in the transport buffer containing $1 \%$ DMSO at final concentration of $100 \mu \mathrm{g} / \mathrm{ml}$. The mixture of all three substances containing propranolol $(100 \mu \mathrm{g} / \mathrm{ml})$, ranitidine $(100 \mu \mathrm{g} / \mathrm{ml})$, and loratadine $(10 \mu \mathrm{g} / \mathrm{ml})$ was also prepared in the transport buffer with 1\% DMSO.

Transport Procedure: On the day of transport study, the culture medium was changed and after $2 \mathrm{~h}$ the transepithelial electrical resistance measured. The monolayers were washed carefully twice in the transport buffer and incubated additionally for $2 \mathrm{~h}$. For the transport from apical to basolateral side (absorption), the inserts were transferred to a new polycarbonate 6-well plate with prewarmed transport buffer in the lower compartment. The drug solution(s) $\left(37^{\circ} \mathrm{C}\right)$ were then added to the top. For basolateral to apical transport (secretion) the drug solutions were added in the lower compartment and the transport medium at the top. Samples $(200 \mu \mathrm{l})$ were taken at $0,15,30,60,90$ and $120 \mathrm{~min}$ from the acceptor compartment and at 0 and $120 \mathrm{~min}$ from the donor compartment. The samples were then analyzed for contents of the drugs. The same transport buffer (with pH 6.6) was used for both apical and basolateral compartments. The experiments were carried out at $37^{\circ} \mathrm{C}$ and the plates were shaken at $55 \mathrm{rpm}$.

Loratadine content was determined by an in-house developed high pressure liquid chromatographic (HPLC) method with mass spectrometric (MS) detection following extraction of the drug. To extract loratadine, aliquots $(50 \mu \mathrm{l})$ of the samples were transferred into glass tubes into which $15 \mu \mathrm{l}$ of $1 \mathrm{M}$ $\mathrm{Na}_{2} \mathrm{CO}_{3}$ solution ( $\mathrm{pH} 10.5$ ) was added and briefly vortexed. Then $250 \mu \mathrm{l}$ of TBME was pipetted into each tube, and after vortexing and shaking for $10 \mathrm{~min}$ at room temperature, the contents of the tubes were centrifuged for $10 \mathrm{~min}$ at $4{ }^{\circ} \mathrm{C}$ $(2000 \boldsymbol{g})$. The centrifuged samples were stored at $-20^{\circ} \mathrm{C}$ for $1 \mathrm{~h}$, and then $200 \mu \mathrm{l}$ of the upper ether layer transferred into Eppendorf tubes to be evaporated to dryness. The dried contents were then reconstituted in $200 \mu \mathrm{l}$ of the mobile phase (acetonitrile: $0.02 \%$ trifluoroacetic acid 40:60, pH 2.66), vortexed and centrifuged for $10 \mathrm{~min}$ at $4{ }^{\circ} \mathrm{C}(3000 \boldsymbol{g})$. Aliquots $(25 \mu \mathrm{l})$ of the sample solutions were then injected onto a Waters Xterra MS $\mathrm{C}_{8}$ column and separation was performed by isocratic elution at room temperature with a flow rate of $0.3 \mathrm{ml} / \mathrm{min}$.

Propranolol was assayed using the HPLC method described in the EP (4th Edition) with minor modification, while ranitidine content was analysed by a slightly modified HPLC method described by Kaliszan et al. ${ }^{8)}$

Calculations: The apparent coefficient of permeation was calculated according to the following equation ${ }^{9}$ :

$$
P_{\text {app }}=d Q / d t /\left(C_{\mathrm{o}}^{*} A\right)(\mathrm{cm} / \mathrm{s})
$$

where, $d Q / d t$ is the linear appearance rate of permeability obtained for the transported amount of the substance over time $(\mu \mathrm{g} / \mathrm{s}), C_{\mathrm{o}}$ is the initial concentration of the drug in the donor compartment $(\mu \mathrm{g} / \mathrm{ml})$, and $A$ is the surface area $\left(\mathrm{cm}^{2}\right)$ of the cell monolayer.

Dissolution Studies The dissolution tests were performed using an automated dissolution testing apparatus VanKel 7010 (VanKel, NC, U.S.A.) attached to a Varian Cary $100 \mathrm{UV}-\mathrm{Vis}$ spectrophotometer and calibrated according to the USP 24 method. Experiments were performed using the USP apparatus 2 with the paddle rotating at $50 \mathrm{rpm}$ (except in FaSSIF) and at $100 \mathrm{rpm}$ in $900 \mathrm{ml}$ of the following media:

- USP simulated gastric fluid (SGF) without pepsin (USP 24/NF 19)

- $0.01 \mathrm{~m}$ hydrochloric acid solution ( $\mathrm{pH} 2.0)$,

$-0.003 \mathrm{~m}$ hydrochloric acid solution ( $\mathrm{pH} 2.5$ ),

$-0.003 \mathrm{M}$ hydrochloric acid solution containing $0.1 \%$ Triton $\mathrm{X} 100$, and

- Fasting State Simulating Intestinal Fluid (FaSSIF) (pH $6.5)^{10)}$

For the experiments using hydrochloric acid solutions, samples were collected automatically every 2 min starting from $2 \mathrm{~min}$ of analysis up to $20 \mathrm{~min}$ and thereafter every $5 \mathrm{~min}$ up to $45 \mathrm{~min}$. The pooled samples were automatically filtered through VanKel's Full-Flow ${ }^{\mathrm{TM}}$ filters $(35 \mu \mathrm{m})$ and the absorbances were measured at wavelength of $277 \mathrm{~nm}$, except for samples containing Triton $\mathrm{X}$ for which $300 \mathrm{~nm}$ was used.

For the FaSSIF experiments, samples were collected manually every $10 \mathrm{~min}$ up to $30 \mathrm{~min}$, then every $15 \mathrm{~min}$ up to $60 \mathrm{~min}$ and thereafter every $30 \mathrm{~min}$ up to $180 \mathrm{~min}$ and every $60 \mathrm{~min}$ up to $720 \mathrm{~min}$ by which time $98 \%$ of loratadine had dissolved. The samples were analyzed using an HPLC method developed in house $\left(\mathrm{C}_{18}\right.$ column with a precolumn, mobile phase: $0.06 \mathrm{M} \mathrm{KH}_{2} \mathrm{PO}_{4}$ buffer:acetonitrile $35: 65$, flow $1.5 \mathrm{ml} / \mathrm{min}$, injection volume $20 \mu \mathrm{l}$ ).

Bioavailability Study in Healthy Volunteers Based on literature information, a steady state study (to minimize intra-subject variability) was performed (as part of a bioequivalency study) by a contract research organization (Algorithm Pharma Inc., Montréal, Canada) after multiple $(\times 6)$ dosing of the drug (in the form of a single tablet containing $10 \mathrm{mg}$ of loratadine) into 13 male volunteers. The 6th dose was orally administered (with $240 \mathrm{ml}$ of drinking water) after overnight fasting to each volunteer and venous blood samples (about $10 \mathrm{ml}$ ) were collected (after the 6th administration) every $15 \mathrm{~min}$ after administration of the drug for $2 \mathrm{~h}$, and thereafter at 2.5, 3, 4, 6, 8, 10, 12, 14, 16 and $24 \mathrm{~h}$. One sample was collected prior to administration of the drug from each volunteer. The content of the drug in the samples was determined by an HPLC method (with MS detection) devel- 
oped by the contract research organization.

In Vitro-In Vivo Correlation (IVIVC) Predicted plasma level versus time profiles were generated by convolution analysis using QWERT software (Version 1.1, SI Computing, UPPSALA, Sweden). No plasma level versus time data are available in the literature for intravenous administration of loratadine, however, mean data after administration of a syrup formulation were obtained through personal communications (Prof. H. Blume) and were used for the weighting function. A dose correction was made in QWERT to compensate for the differences between the in vivo AUCs of the syrup and tablet. The various in vitro dissolution profiles were used as input functions for the convolutions resulting in a series of predicted plasma profiles for comparison with the in vivo data.

\section{RESULTS}

Solubility of Loratadine The solubility profile of loratadine measured in various $\mathrm{pH}$ media within the range of the entire gastrointestinal (GI) tract is presented in Fig. 1. The lowest value for solubility (about $0.004-0.006 \mathrm{mg} / \mathrm{ml}$ ) was obtained in the highest $\mathrm{pH}$ media $(7.5,6.5)$ tested and the highest value of about $4.59 \mathrm{mg} / \mathrm{ml}$ was obtained in $\mathrm{pH} 1.2$ medium. The solubility changed significantly within the well-accepted $\mathrm{pH}$ range of the stomach (fasting/fed state), with a dramatic fall when the $\mathrm{pH}$ was increased from 1.2 to 2 $(1.32 \mathrm{mg} / \mathrm{ml})$, with a further decrease as the $\mathrm{pH}$ was increased to $2.5(0.60 \mathrm{mg} / \mathrm{ml})$.

Dissolution The release profiles of the drug obtained under the low $(50 \mathrm{rpm})$ and high $(100 \mathrm{rpm})$ hydrodynamic conditions in different media are presented in Figs. 2A and $2 \mathrm{~B}$, respectively. Due to the significantly longer dissolution time required to obtain a meaningful amount of release of the drug in FaSSIF medium, the dissolution profiles obtained under FaSSIF conditions are presented separately in Fig. 3.

The rates of dissolution of the drug from the tablets obtained in the lower $\mathrm{pH}$ media were much faster than those obtained in the higher $\mathrm{pH}$ media when compared under similar conditions. The rate of release of the drug dramatically decreased when the $\mathrm{pH}$ of the media was changed from 1.2 to 2. With a further increase in $\mathrm{pH}$ from 2 to 2.5 , the rate of release continued to decrease with higher tablet-to-tablet variations as evidenced from high standard deviation (S.D.) val-

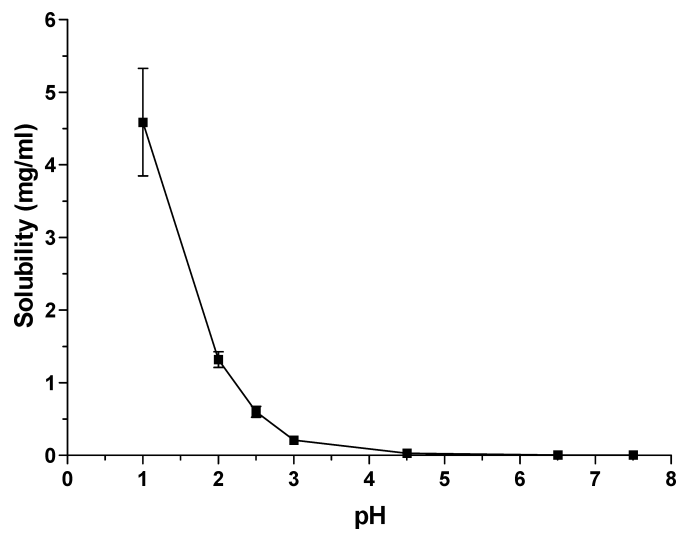

Fig. 1. Solubility Profile of Loratadine Tested in Physiologically Relevant $\mathrm{pH}$ Media of the Gastrointestinal Tract

Vertical bars indicating S.D. values $(n=3)$ are within the points if not visible. ues. At pH 6.5 (FaSSIF medium), the release rate was extremely slow with complete dissolution after about $12 \mathrm{~h}$.

Comparison of the dissolution profiles obtained at 50 and $100 \mathrm{rpm}$ (in the same dissolution medium) clearly demonstrates that the hydrodynamic conditions significantly influence the release profile. The higher the stirring speed was the faster was the rate of release and the lower the variability.

In Vitro Permeability The apparent permeability coefficient $\left(P_{\text {app }}\right)$ obtained for loratadine alone was significantly higher than that found for the mixture of loratadine, propranolol and ranitidine (Table 1), but in both cases the values
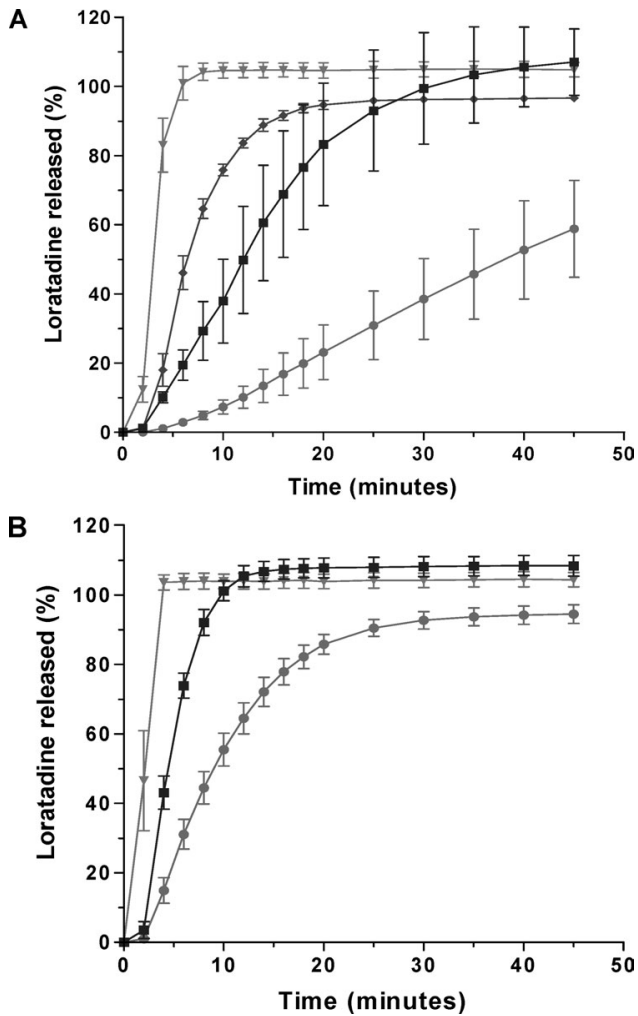

Fig. 2. Comparative Dissolution Profiles of Loratadine from the Tablets in Various Test Media

(A) pH 1.2-SGF ( $)$, pH $2.0(\boldsymbol{\square}), \mathrm{pH} 2.5(\bullet), \mathrm{pH} 2.5$ with Triton $\mathrm{X}(\diamond)$ at $50 \mathrm{rpm}$ and (B) pH 1.2-SGF ( $)$, pH $2.0(\mathbf{\square}), \mathrm{pH} 2.5(\bullet)$ at $100 \mathrm{rpm}$. The tests were performed using the USP paddle method with $900 \mathrm{ml}$ of media at $37 \pm 0.5^{\circ} \mathrm{C}$. Vertical bars indicating S.D. values $(n=6)$ are within the points if not visible.

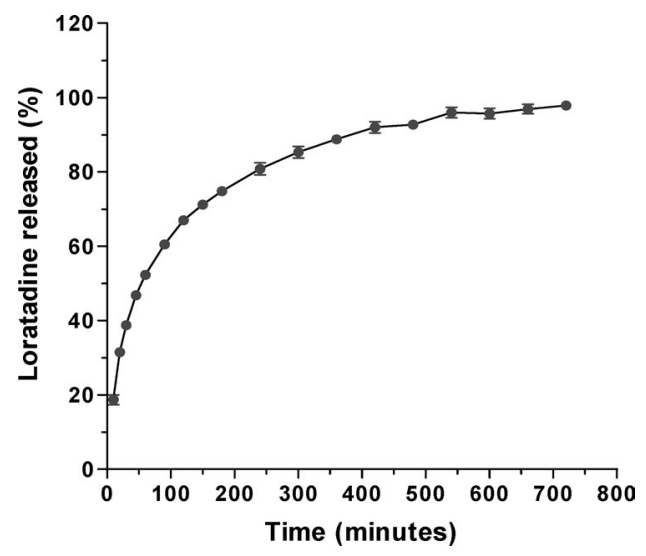

Fig. 3. Dissolution Profile of Loratadine from the Tablets in FaSSIF Medium ( $\mathrm{pH}$ 6.5) Obtained Using the USP Paddle Method with $900 \mathrm{ml}$ of the Medium at $37 \pm 0.5^{\circ} \mathrm{C}$ with Stirring at $100 \mathrm{rpm}$

Vertical bars indicating S.D. values $(n=6)$ are within the points if not visible. 
Table 1. Permeability Coefficients Observed for Loratadine, Propranolol and Ranitidine Separately $\left.{ }^{a}\right)$ and in a Mixture ${ }^{b)}$ of the Three Drugs $(n=3$, except $* n=2)$

\begin{tabular}{lccc}
\hline \hline & & $P_{\text {app }}(\mathrm{cm} / \mathrm{s})$ & \\
\cline { 2 - 4 } Compound & Absorption $^{a)} \pm$ S.D. & Absorption $^{b} \pm$ S.D. & Secretion $^{b} \pm$ S.D. \\
\hline Loratadine & $4.8 \times 10^{-5} \pm 0.7 \times 10^{-5}$ & $2.0 \times 10^{-5} \pm 0.2 \times 10^{-5}$ & $3.1 \times 10^{-5} \pm 0.2 \times 10^{-5}$ \\
Propranolol & $3.8 \times 10^{-5} \pm 0.1 \times 10^{-5}$ & $3.5 \times 10^{-5} \pm 0.1 \times 10^{-5}$ & $3.8 \times 10^{-5} \pm 0.3 \times 10^{-5}$ \\
Ranitidine & $2.7 \times 10^{-7} \pm 0.4 \times 10^{-7}$ & $4.0 \times 10^{-7} \pm 0.2 \times 10^{-7 *}$ & $6.1 \times 10^{-7} \pm 1.8 \times 10^{-7}$ \\
\hline
\end{tabular}

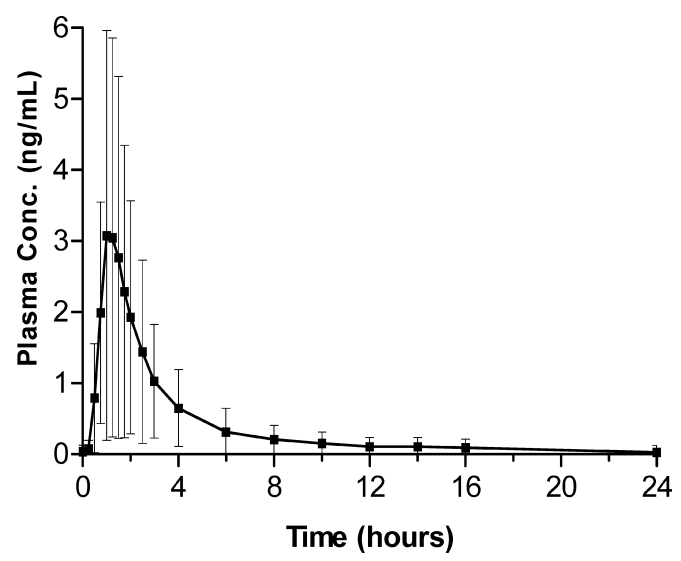

Fig. 4. Plasma Profile after Oral Administration of a Single Tablet of Loratadine $(10 \mathrm{mg})$ at Steady State

The vertical bars indicate S.D. values $(n=13)$ of the plasma levels.

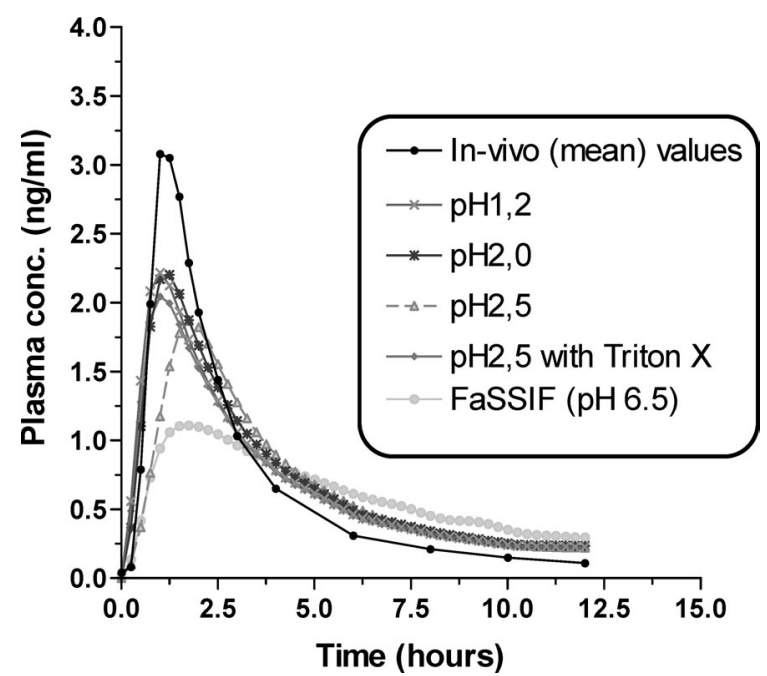

Fig. 5. Plasma Time Profiles Predicted by Convolution of Various in Vitro (Dissolution) Data (Presented Above in Figs. 2A, 3) as Input Functions, Compared with the Observed Mean Plasma Time Profile Obtained from the Bioavailability Study (in Humans)

exceeded $2 \times 10^{-6} \mathrm{~cm} / \mathrm{s}$ suggesting that loratadine would show good oral drug absorption. ${ }^{11)}$

Bioavailability The mean plasma profile obtained after oral administration of a single tablet $(10 \mathrm{mg})$ is presented in Fig. 4. A $C_{\max }$ value of $3.28 \mathrm{ng} / \mathrm{ml}$ was reached at $1 \mathrm{~h}\left(t_{\max }\right)$ with an $A U C_{t}$ value of $8.8 \mathrm{ng} \cdot \mathrm{h} / \mathrm{ml}$. The standard deviations obtained for the plasma concentrations throughout the sampling points were very high with coefficients of variation (CV) ranging from 78 to $120 \%$ at times up to $12 \mathrm{~h}$ after administration of the drug and reaching $273 \%$ at the $24-\mathrm{h}$ test point.

In Vivo Absorption and IVIVC Predicted plasma time

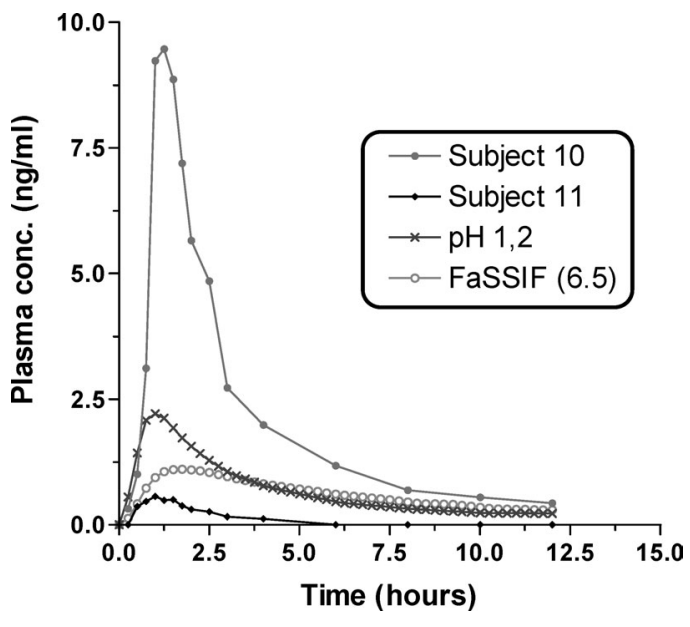

Fig. 6. Predicted Plasma Time Profiles from in Vitro Release Profiles Using pH 1.2 and FaSSIF (pH 6.5) Dissolution Media, Compared with the Extremes of Plasma Profiles Obtained from the Bioavailability Study (in Humans)

profiles obtained by convolution of various in vitro dissolution data sets (as input functions) are shown in Fig. 5. All the curves predicted from the dissolution data at $\mathrm{pH} 1.2,2.0$, and 2.5 (with and without Triton) are in reasonable agreement with the mean plasma time profile from the bio-study; but the dissolution data set for FaSSIF is a poor predictor of the mean in vivo profile (Fig. 5).

There was high intersubject variability in the biostudy as shown by the large error bars in Fig. 4; hence, when plasma time profiles for the individuals with the lowest and highest $A U C$ s are compared with the convoluted profiles, it is apparent that there is little evidence to recommend one dissolution medium over another for predictive purposes (Fig. 6).

\section{DISCUSSION}

A drug is considered highly soluble when the highest dose is soluble in $250 \mathrm{ml}$ or less of water over the $\mathrm{pH}$ range of the gastrointestinal tract ( $\mathrm{pH} 1-7.5)$. Loratadine is readily soluble at low $\mathrm{pH}$ values but at $\mathrm{pH}$ values of the upper range it would not meet the definition of "highly soluble" (Fig. 1). Consequently, loratadine with low solubility would be classified as either Class II (high permeability) or IV (low permeability) in the BCS.

A drug substance is defined as highly permeable when the extent of absorption in humans is $\geq 90 \%$ of an administered dose, based on mass-balance or in comparison to an intravenous reference dose; however, there are no intravenous data available for loratadine. Grès et al. ${ }^{11)}$ have explored the relationship between oral drug absorption in humans and the permeability of drugs across Caco-2 monolayers. They re- 
ported that if the apparent permeability coefficient was at least $2 \times 10^{-6} \mathrm{~cm} / \mathrm{s}$, then $100 \%$ absorption could be expected in humans. So the permeability of loratadine across Caco-2 monolayers was explored in this study.

The $P_{\text {app }}$ values obtained for propranolol are in agreement with values reported in the literature ${ }^{11)}$ for this highly permeable compound, but for ranitidine the values reported in the literature $^{12)}$ are slightly higher than what we obtained, which is probably attributable to analytical precision and accuracy due to its very low permeability. Loratadine added to the mixture with propranolol and ranitidine appeared to have significantly lower permeability than when studied alone suggesting interference of either propranolol or ranitidine or both these compounds with permeability of loratadine. It has been reported that loratadine is a P-gp substrate, ${ }^{13)}$ and there are also reports about ranitidine to act as a P-gp substrate ${ }^{14,15}$ ) although there is a contradicting report. ${ }^{13}$ ) The presence of the two possible P-gp substrates in the same system might have interfered with the permeability of each other causing the observed difference.

In a separate experiment, we demonstrated that the transportation of loratadine is not concentration dependent (within the range of $2.5-10.0 \mu \mathrm{g} / \mathrm{ml}$ ) (data not shown) suggesting that the transportation process is not carrier-mediated.

Irrespective of the conditions of the experiments (either in mixture or alone), the permeability of loratadine exceeded the lower limit (Table 1) suggested by Grès et al. ${ }^{11)}$ indicating that loratadine should be classified as a Class II, low soluble, highly permeable drug.

Class II drugs are generally known to be problematic in terms of their dissolution behaviors, and selection of appropriate dissolution method(s) and media for predicting their bioavailability seems to be challenging. Since dissolution is the rate limiting step for absorption and bioavailability of this class of drugs, it seems feasible to select appropriate dissolution media and conditions to establish IVIVC, and such methods have been recommended in literature. ${ }^{10,16)}$

The challenging nature of selecting appropriate dissolution method for predicting absorption and bioavailability is evident from the dissolution data and IVIVC we obtained here. As expected, the fastest in vitro rate of release of the drug from the tablets was obtained in SGF ( $\mathrm{pH} 1.2$ ), which is a reflection of the highest solubility of the drug at this $\mathrm{pH}$. The dramatic fall in the rate of release of the drug in $\mathrm{pH} 2.0$ medium was also anticipated since the solubility also decreases in a similar fashion (see Fig. 1) when the $\mathrm{pH}$ was increased from 1.2 to 2 . Further decrease in rate of release of the drug with increase in $\mathrm{pH}$ of the dissolution media is in agreement with its solubility profile. Such changes in the dissolution profiles of the drug within the expected $\mathrm{pH}$ range of the stomach was anticipated to be one cause of high individual variability during in vivo release of the drug among individuals since the $\mathrm{pH}$ of the stomach is known to differ significantly among individuals ${ }^{17)}$ and is within the range of 1 to $3^{18)}$ or even higher, 1 to $5^{19)}$ under fasting conditions. Furthermore, the very slow dissolution rate in the FaSSIF suggests that if undissolved loratadine is emptied from the stomach, the absorption rate will be dramatically slower compared with those individuals where complete dissolution occurs in the stomach. In fact, the gastric emptying rate can have a significant impact on the absorption profile of the drug since the movement of drugs (both in solution and in dosage form) are effected by various levels of motility of the GI tract, known to have four different phases called the "migrating motor complex", each phase performing different functions taking significantly different lengths of time for completion. The gastric emptying rate, therefore, can vary according to the phase of the GI motility at the time the drug was taken. ${ }^{19)}$ During the in vitro studies, the stirring rate had a pronounced effect on the extent and variability of the dissolution (Figs. 2A, 2B) further suggesting that differences in GI motility conditions among individuals may be contributing to the variability in the bioavailability data.

Our bioavailability data with significantly high $\mathrm{CV}$ values clearly demonstrate the high intersubject variability of the pharmacokinetic parameters of loratadine, which is in line with other reports. ${ }^{5)}$ Although post-absorption differences may contribute to the intersubject variability, our solubility and dissolution profile data for the drug in biologically relevant media suggest that these properties are, at least in part, responsible for the variable in vivo behavior. Therefore, inclusion of high number of volunteers in bioequivalence studies for loratadine generic products seems to be unavoidable in order to meet the required bioequivalence criteria of $90 \%$ confidence limit.

Attempts to predict in vivo dissolution using the bioavailability data as the input function were unsuccessful due to the instability of the deconvolution process. ${ }^{20)}$ The time difference between the mean $t_{\max }$ values for the syrup and the tablet plasma data was very small, suggesting that the tablet dissolution may have occurred rapidly in vivo, at least on average. Rather than pursuing various approaches, which have been suggested to stabilize deconvolution, we used convolution to predict plasma levels which could then be compared with the in vivo data obtained in humans. The agreement between the predicted profiles and mean plasma profile from the bio-study is reasonable (Fig. 5) for the low $\mathrm{pH}$ dissolution data, especially given that the weighting data came from a different panel of volunteers. This suggests that low $\mathrm{pH}$ dissolution media, representing the upper region of the GI tract, are more bio-relevant than FaSSIF. Previous reports have noted that for drugs like loratadine, a week base, dissolution testing under fasting state simulated conditions is of paramount importance. ${ }^{10,21)}$

Although the convoluted plasma levels using low $\mathrm{pH}$ dissolution data were in reasonable agreement with the mean data from the biostudy, it was not possible to choose appropriate dissolution media when individual biodata were considered (Fig. 6). If the intersubject variability relates in large part to dissolution occurring in different regions of the GI tract for different individuals as discussed above, dissolution testing in FaSSIF has also some merits.

\section{CONCLUSION}

The data presented here demonstrate low solubility and high permeability of loratadine and put the drug under Class II according to BCS. The in vitro dissolution data obtained for the studied formulation correlate well with the solubility profile of the drug in various $\mathrm{pH}$ media within the GI tract, and provide an insight into the high variability of the pharmacokinetic data obtained from the bioavailability study. 
Evaluation of both the in vitro and in vivo data demonstrates reasonable agreement between the two sets of data; particularly, the results of the in vivo study in healthy volunteers i.e., quick absorption and good bioavailability, are supported by the data obtained from the permeability study demonstrating the usefulness of the Caco- 2 cell model for such purposes.

The IVIVC suggests that while the use of low $\mathrm{pH}$ dissolution media, rather than FaSSIF, is more predictive of average in vivo behaviour, using FaSSIF as a medium, in addition to the low $\mathrm{pH}$ media, is relevant given that in some individuals, dissolution may occur in the intestine, and the gastric emptying rate would have an impact on absorption of the drug.

Acknowledgements We would like to thank Ms. Dubravka Moskatelo and Ms. Dubravka Županić for their assistance in maintaining the Caco-2 cell cultures, Ms. Mira Šelendić and Mr. Ivica Kušer for their technical assistance in preparing samples for HPLC-MS analysis, Mrs. Snježana Vodopija-Mandić and Ms. Snježana Jurlina for performing the dissolution study using FaSSIF medium, and Ms. Vesna Munić, Ms. Martina Bosnar and Ms. Vesna Eraković for their fruitful discussions and help in designing the permeability studies. The helpful comments and advice of Prof. Ian Tucker of the University of Otago (New Zealand) and Dr. Christos Reppas of National and Kapodistrian University of Athens (Greece) in preparing the manuscript are appreciated.

\section{REFERENCES AND NOTES}

1) Present address: Veterinarska stanica Čakovec, Rudolfa Steinera 7, 40000 Čakovec, Croatia.

2) Present address: Technology Improvement. Product Supply, PLIVA Croatia, Prilaz baruna Filipovića 25, 10000 Zagreb, Croatia.

3) Hilbert J., Radwanski E., Weglein R., Luc V., Perentesis G., Symchowicz S., Zampaglione N., J. Clin. Pharmacol., 27, 694-698 (1987).
4) Katchen B., Cramer J., Chung M., Ann. Allergy, 55, 393 (1985).

5) Radwanski E., Hilbert J., Symchowicz S., Zampaglione N., J. Clin. Pharmacol., 27, 530-533 (1987).

6) Amidon G. L., Lennernäs H., Shah V. P., Crison J. R., Pharm. Res., 12, 413-420 (1995).

7) CDER, Guidance for Industry. Waiver of in vivo bioavailability and bioequivalence studies for immediate-release solid dosage forms based on Biopharmaceutics Classification System. Rockville: FDA. (August 2000).

8) Kaliszan R., Nasal A., Turowski M., Biomed. Chromatogr., 9, 211 215 (1995).

9) cited in: Gao J. G., Hugger E. D., Beck-Westernmeyer M. S., Borchart R. T., "Estimating intestinal mucosal permeation of compounds using Caco-2 cell monolayers," "Current Protocols in Pharmacology," ed. by Enna S. J., Williams M., Ferkany J. W., Kenakin T., Porsolt R. D., Sullivan J. P., John Wiley \& Sons, Inc., New York, 2000, pp. 7.2.1.7.2.23.

10) Galia E., Nicolaides E., Hörter D., Löbenberg R., Reppas C., Dressman J. B., Pharm. Res., 15, 698-705 (1998).

11) Grès M.-C., Julian B., Bourrié M., Meunier V., Roques C., Berger M., Boulenc X., Berger Y., Fabre G., Pharm. Res., 15, 726-733 (1998).

12) Rege B. D., Yu L. X., Hussain A. S., Polli J. E., J. Pharm. Sci., 90, 1776-1786 (2001).

13) Polli J. W., Wring S. A., Humphreys J. E., Huang L., Morgan J. B., Webster L. O., Serabjit-Singh C. S., J. Pharmacol. Exp. Ther., 299, $620-628$ (2001).

14) Lentz K. A., Polli J. W., Wring S. A., Humphreys J. E., Polli J. E., Pharm. Res., 17, 1456-1460 (2000).

15) Lee K., Chee N., Brouwer K. L. R., Thakker D. R., J. Pharmacol. Exp. Ther, 303, 574-580 (2002).

16) Galia E., Horton J., Dressman J. B., Pharm. Res., 16, 1871-1875 (1999).

17) Evans D. F., Pye G., Bramley R., Clark A. G., Dyson T. J., Hardcastle J. D., Gut, 29, 1035-1041 (1988).

18) Dressman J. B., Amidon G. L., Reppas C., Shah V. P., Pharm. Res., 15, $11-22$ (1998).

19) cited in: Golub A. L., Frost R. W., Betlach C. J., Gonzalez M. A., J. Allergy Clin. Immunol., 78, 689-694 (1986).

20) Langenbucher F., Pharm. Ind., 44, 1166-1172 (1982).

21) Kostewicz E. S., Brauns U., Becker R., Dressman J. B., Pharm. Res., 19, 345-349 (2002). 